\title{
Laser Therapy in the Treatment of Peri-Implantitis: State-of-the-Art, Literature Review and Meta-Analysis
}

\author{
Massimo Pisano ${ }^{\dagger}$, Alessandra Amato, Pasquale Sammartino, Alfredo Iandolo, Stefano Martina (D) and \\ Mario Caggiano *, + (D)
}

Citation: Pisano, M.; Amato, A.; Sammartino, P.; Iandolo, A.; Martina, S.; Caggiano, M. Laser Therapy in the Treatment of Peri-Implantitis: State-of-the-Art, Literature Review and Meta-Analysis. Appl. Sci. 2021, 11, 5290 .

https://doi.org/10.3390/app11115290

Academic Editor: Hom-Lay Wang

Received: 2 May 2021

Accepted: 3 June 2021

Published: 7 June 2021

Publisher's Note: MDPI stays neutral with regard to jurisdictional claims in published maps and institutional affiliations.

Copyright: (c) 2021 by the authors. Licensee MDPI, Basel, Switzerland. This article is an open access article distributed under the terms and conditions of the Creative Commons Attribution (CC BY) license (https:// creativecommons.org/licenses/by/ $4.0 /)$.
Department of Medicine, Surgery and Dentistry, Scuola Medica Salernitana, University of Salerno, 84084 Salerno, Italy; pisano.studio@virgilio.it (M.P.); aamato@unisa.it (A.A.); pasqualesammartino91@gmail.com (P.S.); aiandolo@unisa.it (A.I.); smartina@unisa.it (S.M.)

* Correspondence: macaggiano@unisa.it

+ These Authors contributed equally to this paper.

Featured Application: The treatment of the peri-implantitis is still challenging, and no consensus was found in the literature on which is the best treatment protocol. Following the results of our meta-analysis, the use of dental laser does not offer statistically significant improvements in terms of PPD reduction and CAL gain if compared to conventional mechanical therapy.

Abstract: (1) Background: The treatment of the peri-implantitis is still challenging, and no consensus was found in the literature on which is the best treatment protocol. In recent years, numerous authors have proposed the use of the dental laser as an alternative and effective method for decontaminating the surface of infected implants. Therefore, the aim of this work was to examine the state-of-the-art on the use of lasers in the treatment of peri-implantitis through the literature. (2) Methods: An electronic search was conducted through the PubMed database; we selected and reviewed articles that evaluated the effects of laser irradiation in the treatment of peri-implantitis. (3) Results: The use of lasers seems to provide similar results if compared with conventional mechanical therapy. The included studies were divided into three groups based on the active medium of the laser used for the treatments being tested (Erbium: Yttrium Aluminum Garnet, $\mathrm{CO}_{2}$ and diode laser). (4) Conclusions: The lasers showed positive results, on average, after 6 months of follow-up. Comparative clinical trials conducted with the aim to test the efficiency of laser irradiation, in addition to conventional therapy, indicate that this technique can induce similar results if compared to conventional therapy alone.

Keywords: laser therapy; peri-implantitis; dental implants; laser; implant failure

\section{Introduction}

Dental Implantology is, nowadays, a technique with highly predictable results and, for this reason, represents a preponderant part of the range of therapeutic alternatives in the treatment of partially and totally edentulous patients [1].

The application of implant-supported prosthetics in dentistry began towards the end of the 1980s, even if their technical development occurred at the beginning of 1960, when Branemarck's group introduced new and revolutionary concepts deriving from the knowledge of the biological phenomena that occurred in the interface between bone and implant. The term of osseointegration was introduced to define the formation of strict and functional link between bone and implant fixture, subjected to prosthetic loading, without connective tissue interposition [2]. However, dental implants over time can present pathological changes in the surrounding tissues, which can cause osseointegration defects. These pathological conditions, indicated by the term "mucositis" and "peri-implantitis" (PI) (respectively, if the inflammatory processes affect only the marginal gingiva or the deep peri-implant tissues), have increased in recent years, in proportion to the grater insertion of the dental implant. 
Peri-implantitis (PI) concerns complex phlogistic phenomena that occur in the tissues surrounding a functionally active implant. A bad balance between pathogenic bacterial load and host response could trigger an inflammatory cascade that would first affect the superficial peri-implant soft tissues (mucositis) and then progress into the deep layers, with a loss of implant support bone which can be clinically and radiologically highlighted (peri-implantitis) [3].

PI is therefore an infectious disease in which the bacteria colonizing the dental implant's surface and participating in the development of the biofilm are responsible for the genesis of an inflammatory response and inhibiting the integration of bone cells to the surface of the implant [4,5].

Clinical diagnosis of PI is based on the following findings: bleeding on light periimplant probing, an increase in probing depth, and marginal bone loss (however, subsequent to the physiological remodeling that is observed following the placement and the loading of the dental implants) [6].

Based on a review article published by Mombelli and his co-worker, the prevalence of PI was rated in the order of $10 \%$ of implants and $20 \%$ of patients $5-10$ years after implant loading $[7,8]$. However, the authors also acknowledge that this statement derives from the analysis of clinical studies reporting rather variable and difficult-to-compare data, and for this reason, it is not definitively representative of the diffusion of PI among patients.

Peri-implant treatment is still challenging; although several treatments have been proposed, no consensus on which is the most predictable, reproducible and effective procedure has been reported in the literature.

In the treatment of PI, it is mandatory not only to remove all the inflammatory soft tissue around the peri-implant pocket and eventually apply bone augmentation techniques, but also to decontaminate the infected fixtures parts. Several approaches for implant decontamination aimed to eliminate bacterial biofilm are noted: mechanical and pharmacological decontamination, implantoplasty and laser irradiation. However, none of the curative protocols reported in the literature has proven to be effective in the treatment of PI [9].

Therapeutic performances of mechanical or chemical modalities seem to be limited due to resistant bacterial strains, limited access to inflamed area and pharmacologic limitations such as in-site drug dosage or insufficient antibacterial effect. Moreover, mechanical strategies such as metallic curettes, ultrasonic metal tip scalers and air powder abrasion may develop a roughened implant surface, which itself increases bacterial colonization and biofilm formation [10]. Recently, a noticeable tendency has urged scientists toward application of lasers in order to decontaminate infected implants parts and peri-implant inflamed areas due to selective calculus removal, bactericidal and hemostatic effects of lasers. In vitro evaluations have demonstrated the efficacy of Erbium-doped Yttrium Aluminum Garnet (Er:YAG), carbon dioxide laser $\left(\mathrm{CO}_{2}\right)$ and diode lasers to effectively remove bacterial biofilm from titanium disks [11]. Moreover, microscopic evaluations have ensured that proper application of these lasers do not disturb the titanium implant fixture.

The aim of our work was to rate, through an updated literature review, the real effectiveness of dental laser used in the treatment of PI.

\section{Materials and Methods}

An electronic search was conducted through the PubMed database (http:/ / www.ncbi. nlm.nih.gov/pubmed/ accessed on 15 November 2020); we selected and reviewed articles that involved the role and the effectiveness of dental-laser use in PI treatment. The research was carried out by using the following keywords: "lasers" OR "laser"; "periimplantitis" OR "peri-implantitis"; "periimplant disease" OR "peri-implant disease"; "periimplant infection" OR "peri-implant infection"; and "photodynamic therapy", "Implant surface decontamination", "Implant failure" and "dental implant". In addition, the bibliography of the selected articles was also examined in order to find any further publication of interest for the aim of this study. Bibliographic research was conducted independently by two 
different authors (MP and MC); abstracts were reviewed to identify those relevant to the aims of the present study, for which the full papers were then obtained.

\subsection{Eligibility Criteria}

- Inclusion criteria:

o Population: Implants affected by mucositis and peri-implantitis in human;

o Study design: Randomized-controlled trial, case-control studies, cases series with a minimum of 3 patients, meta-analysis conducted on "in vivo" studies;

o Language: Papers written in English language only;

o Time of publication: Paper published in the last 15 years;

o Treatment: Any laser-aided treatment;

o Follow-up: Studies followed by an observational period of at least 3 months.

- Exclusion criteria:

o Studies assessing the effectiveness of laser treatment using only radiographs;

o Studies evaluating the effectiveness of lasers in peri-implantitis treatment in vitro only.

\subsection{Data Analysis}

A meta-analysis was conducted with Epidat 3.1 software (Xunta de Galicia, Spain) in random effect mode, to compare the relevant outcomes obtained in the studies included in our review with the aim to establish the role of laser irradiation in the peri-implantitis therapy. The publication bias was evaluated by using a Funnel plot and the Egger test for the main periodontal clinical changes (Periodontal Probing Depth reduction and Clinical Attachment Level gain). A sensitivity analysis of the meta-analysis results was also performed for these outcomes. The statistical heterogeneity among studies was assessed by using the Dersimonian and Laird's heterogeneity test, based on $Q$ statistic (chi-square), in order to know the percentage of variation in the global estimate that was attributable to heterogeneity.

\section{Results}

The literature search has widely confirmed that, in order to achieve the recovery of peri-implant tissue health, it is essential to remove the inflammatory tissue and the bacteria from the infected titanium surface. Among the various decontamination protocols proposed, those that involve the use of lasers seem to provide no statistically significant differences, in terms of PPD reduction and CAL gain, if compared with conventional mechanical therapy.

The initial electronic search resulted in the identification of over 200 potential papers. Article, title and abstract screening was conducted, and, according to the established inclusion criteria, only 60/200 were selected. After examining the full-text articles, we found that $36 / 60$ met the inclusion criteria, and they were finally chosen to be included in this review (Figure 1).

The included studies were subsequently divided into three groups based on the active medium of the laser used for the treatments being tested. Specifically, clinical evaluation studied peri-implant disease treatment through the use of the Erbium: Yttrium Aluminum Garnet (Er:YAG) laser was included in Group 1, $\mathrm{CO}_{2}$ laser in Group 2 and diode laser in Group 3.

For analytical purposes, for each study, we noted the following: the first author and the publication year, the type of the used laser and its settings, the number of patients treated, the study design, the duration of follow-up, the results obtained with the laser therapy and the conclusion resulting from the findings of the authors. These data are summarized in Tables 1-3. A meta-analysis was conducted on three studies in the Group 1 (Schwarz 2004, Schwarz 2013, Wang 2020) to evaluate PPD reduction (Figure 2) and CAL gain (Figure 3) after treatment. The results obtained showed no significant differences between patients treated with Er:YAG laser irradiation if compared at patients treated 
with conventional mechanical therapy alone. Similar results were found in Group 3 after conducting a meta-analysis on three studies (Schar 2013, Bassetti 2013, Tenore 2020) to compare the PPD reduction obtained in patients treated with diode laser irradiation vs. conventional mechanical therapy (Figure 4). No significant heterogeneity (Figure 5) or publication bias (Figures 6 and 7) was observed when combining all controlled studies for the outcomes tested $(p>0.005)$.
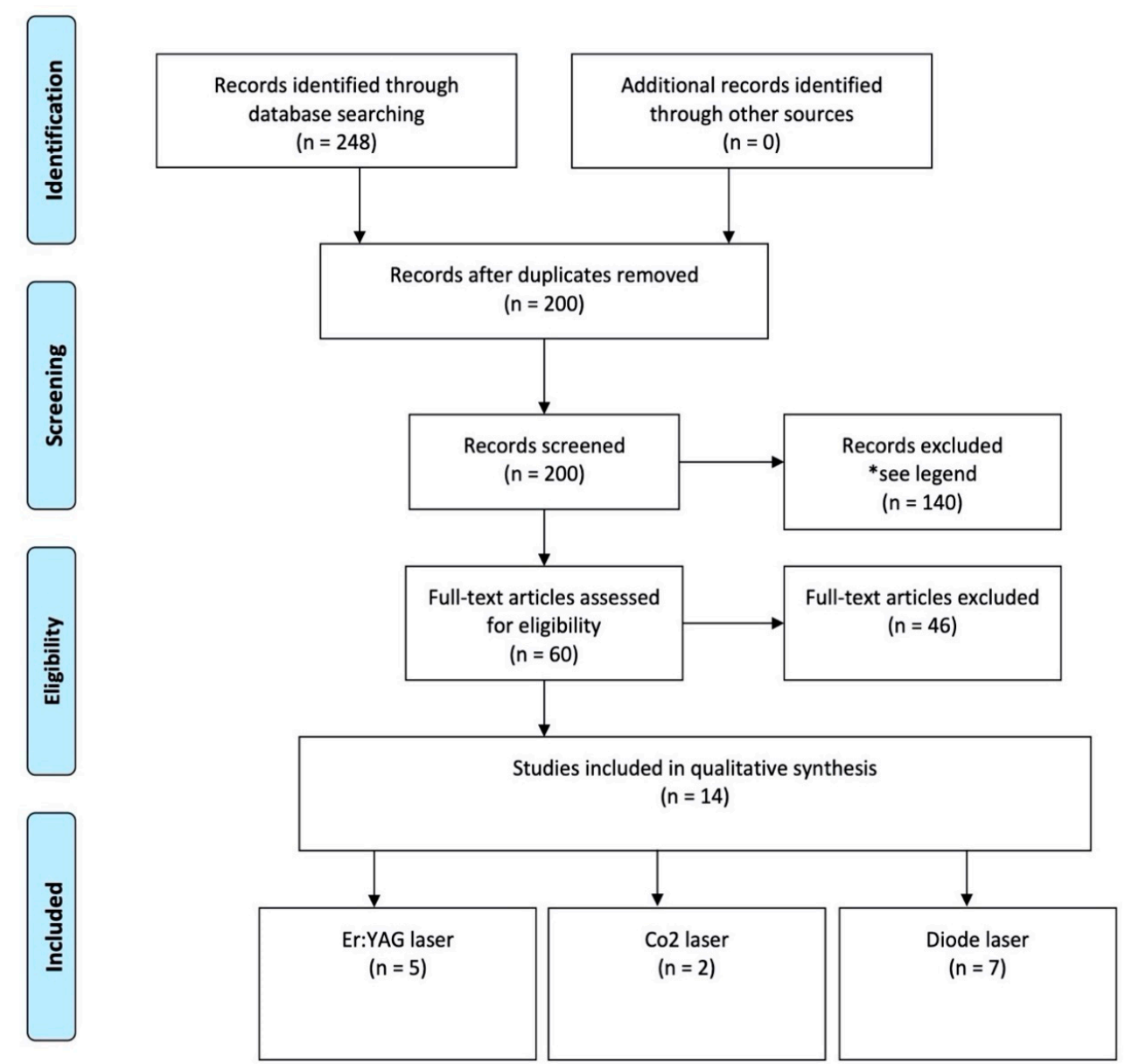

Figure 1. Flowchart depicting the article selection process. * Exclusion criteria: studies assessing the effectiveness of laser treatment by using only radiographs and evaluating the effectiveness of lasers in peri-implantitis treatment in vitro only.

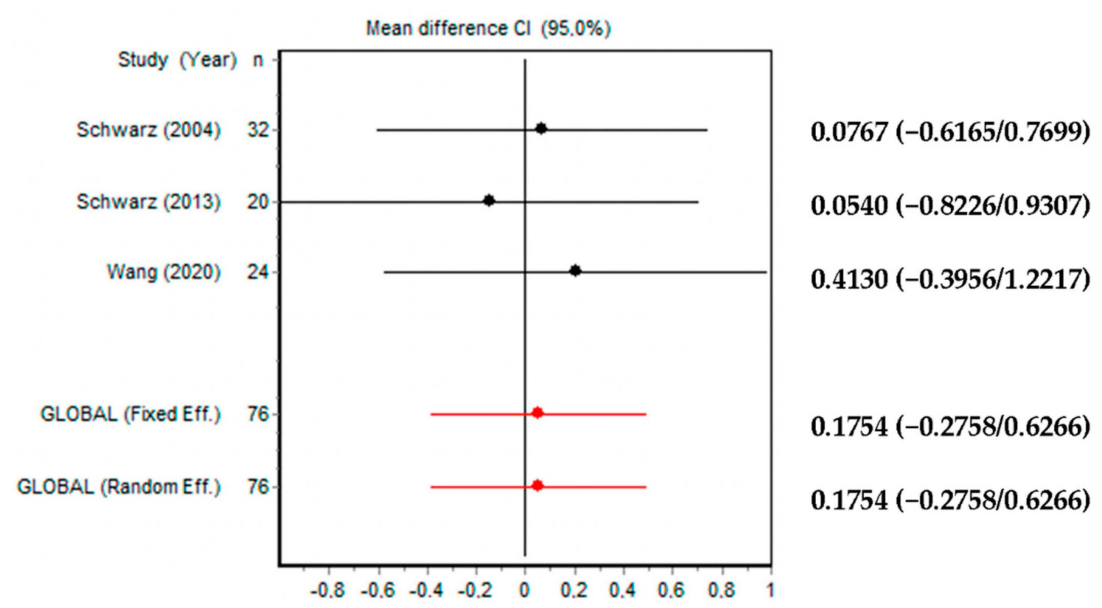

Figure 2. Forest plot for CAL gain mean (Er:YAG laser vs. conventional mechanical therapy). 
Table 1. Use of Er:YAG laser in peri-implantitis treatment.

\begin{tabular}{|c|c|c|c|c|c|c|c|}
\hline $\begin{array}{l}\text { First } \\
\text { Author- } \\
\text { Year }\end{array}$ & $\begin{array}{c}\text { Type of } \\
\text { Laser }\end{array}$ & Laser Settings & No. of Patients & Study Design & $\begin{array}{c}\text { Follow-Up } \\
\text { Duration }\end{array}$ & $\begin{array}{l}\text { Results } \\
\text { Obtained at the } \\
\text { End of the } \\
\text { Follow-Up }\end{array}$ & Conclusions \\
\hline $\begin{array}{l}\text { Schwarz } \\
\text { et al., } \\
2005 \text { [12] }\end{array}$ & Er:YAG & $\begin{array}{l}\text { Wavelength } \\
2.94 \mathrm{~nm} \text { at } 100 \\
\mathrm{~mJ} / \text { pulse }\end{array}$ & $\begin{array}{l}20 \text { patients with a } \\
\text { total of } 32 \text { dental } \\
\text { implants. Test group: } \\
16 \text { implants; Control } \\
\text { group: } 16 \text { implants }\end{array}$ & $\begin{array}{l}\text { Pilot study. Test } \\
\text { group: Er: YAG laser } \\
\text { instrumentation. } \\
\text { Control group: } \\
\text { mechanical } \\
\text { debridement using } \\
\text { plastic curettes and } \\
\text { antiseptic therapy } \\
\text { with chlorhexidine } \\
\text { digluconate }(0.2 \%)\end{array}$ & 6 months & $\begin{array}{l}\text { Test group: mean } \\
\text { value of BOP } \\
\text { decreased from } \\
83 \% \text { at baseline } \\
\text { to } 31 \% \text { after } 6 \\
\text { months. A mean } \\
\text { CAL change } \\
\text { from } 5.8 \pm 1 \mathrm{~mm} \\
\text { at baseline to } 5.1 \\
\pm 1.1 \text { mm after } 6 \\
\text { months. Control } \\
\text { group: mean } \\
\text { value of BOP } \\
\text { decreased from } \\
80 \% \text { at baseline } \\
\text { to } 58 \% \text { after } 6 \\
\text { months. A mean } \\
\text { CAL change } \\
\text { from } 6.2 \pm 1.5 \\
\text { mm at baseline } \\
\text { to } 5.6 \pm 1.6 \text { mm } \\
\text { after } 6 \text { months }\end{array}$ & $\begin{array}{l}\text { After } 6 \text { months, } \\
\text { the difference in } \\
\text { CAL gain } \\
\text { between the two } \\
\text { groups was } \\
\text { statistically not } \\
\text { significant ( } p> \\
0.05) \text {. A } \\
\text { statistically } \\
\text { significant higher } \\
\text { reduction of BOP } \\
\text { than in control } \\
\text { group was } \\
\text { achieved in the } \\
\text { test group }\end{array}$ \\
\hline $\begin{array}{l}\text { Renvert } \\
\text { et al., } \\
2011 \text { [13] }\end{array}$ & Er:YAG & $100 \mathrm{~mJ} /$ pulse & $\begin{array}{l}42 \text { patients. Test } \\
\text { group: } 21 \text { patients; } \\
\text { Control group: } 21 \\
\text { patients }\end{array}$ & $\begin{array}{l}\text { Randomized } \\
\text { controlled trial. Test } \\
\text { group: implants were } \\
\text { treated with Er:YAG } \\
\text { laser instrumentation. } \\
\text { Control group: } \\
\text { implants were } \\
\text { treated with } \\
\text { air-polishing used in } \\
\text { the pocket around } \\
\text { the implant }\end{array}$ & 6 months & $\begin{array}{l}\text { The mean } \\
\text { probing depth } \\
\text { (PPD) reductions } \\
\text { in the control } \\
\text { and test groups } \\
\text { were } 0.9 \mathrm{~mm} \text { and } \\
0.8 \mathrm{~mm} \text {, with } \\
\text { mean bone-level } \\
\text { changes (loss) of } \\
-0.1 \mathrm{~mm} \text { and } \\
-0.3 \mathrm{~mm}, \\
\text { respectively }\end{array}$ & $\begin{array}{l}\text { No statistically } \\
\text { different results } \\
\text { were achieved } \\
\text { between control } \\
\text { and test group } \\
\text { after } 6 \text { months }\end{array}$ \\
\hline $\begin{array}{l}\text { Schwarz } \\
\text { et al., } \\
2013 \text { [14] }\end{array}$ & Er:YAG & $\begin{array}{l}\text { Wavelength } \\
2.94 \mathrm{~nm} \\
\text { at } 100 \\
\mathrm{~mJ} / \text { pulse }\end{array}$ & $\begin{array}{c}17 \text { patients. Test } \\
\text { group: } 7 \text { patients; } \\
\text { Control group: } 10 \\
\text { patients }\end{array}$ & $\begin{array}{c}\text { Randomized } \\
\text { controlled trial. In } \\
\text { both groups surgical } \\
\text { therapy to obtain a } \\
\text { complete granulation } \\
\text { tissue remotion and } \\
\text { implantoplasty at } \\
\text { exposed fixtures } \\
\text { parts were } \\
\text { performed. } \\
\text { Subsequently, } \\
\text { implants were } \\
\text { treated with Er:YAG } \\
\text { laser in the test } \\
\text { group, while only } \\
\text { with plastic curettes } \\
\text { in the control group }\end{array}$ & 48 months & $\begin{array}{c}\text { In control group } \\
\text { a higher } \\
\text { reduction in } \\
\text { mean BOP } \\
\text { (control: } 85.2 \pm \\
16.4 \% \text { versus test: } \\
71.6 \pm 24.9 \% \text { ) } \\
\text { and better CAL } \\
\text { values (control: } \\
1.5 \pm 2.0 \text { mm } \\
\text { versus test: } 1.2 \pm \\
2.0 \text { mm) was } \\
\text { obtained when } \\
\text { compared with } \\
\text { the test group }\end{array}$ & $\begin{array}{l}\text { Implants } \\
\text { surfaces decon- } \\
\text { taminations } \\
\text { methods did not } \\
\text { influence clinical } \\
\text { outcome } \\
\text { obtained with } \\
\text { surgical therapy } \\
\text { in the treatment } \\
\text { of advanced } \\
\text { peri-implantitis } \\
\text { disease }\end{array}$ \\
\hline $\begin{array}{l}\text { Pommer } \\
\text { et al., } \\
2016 \text { [15] }\end{array}$ & Er:YAG & $\begin{array}{l}\text { Wavelength } \\
2.94 \mathrm{~nm}\end{array}$ & $\begin{array}{c}142 \text { patients. Group 1: } \\
72 \text { patients, Group 2: } \\
47 \text { patients; Group 3: } \\
23 \text { patients }\end{array}$ & $\begin{array}{l}\text { Comparative study. } \\
\text { Group 1: laser } \\
\text { decontamination; } \\
\text { Group 2: } \\
\text { implantoplasty; } \\
\text { Group 3: laser } \\
\text { decontamination + } \\
\text { implantoplasty }\end{array}$ & 9 years & $\begin{array}{l}\text { Success rate / } \\
\text { implant failure } \\
\text { after 9-years of } \\
\text { follow-up. Laser } \\
\text { therapy: } 91.9 \% \\
\text { success rate; } \\
\text { Implantoplasty: } \\
\text { 87.2\% success } \\
\text { rate; } \\
\text { Combination of } \\
\text { both technique: } \\
82.6 \% \text { success } \\
\text { rate }\end{array}$ & $\begin{array}{l}\text { No statistically } \\
\text { difference was } \\
\text { found by the } \\
\text { authors after } 9 \\
\text { years of } \\
\text { follow-up } \\
\text { between } 3 \\
\text { groups }\end{array}$ \\
\hline
\end{tabular}


Table 1. Cont.

\begin{tabular}{|c|c|c|c|c|c|c|c|}
\hline $\begin{array}{l}\text { First } \\
\text { Author- } \\
\text { Year }\end{array}$ & $\begin{array}{l}\text { Type of } \\
\text { Laser }\end{array}$ & Laser Settings & No. of Patients & Study Design & $\begin{array}{l}\text { Follow-Up } \\
\text { Duration }\end{array}$ & $\begin{array}{c}\text { Results } \\
\text { Obtained at the } \\
\text { End of the } \\
\text { Follow-Up }\end{array}$ & Conclusions \\
\hline $\begin{array}{c}\text { Wang } \\
\text { et al., } \\
2020 \text { [16] }\end{array}$ & Er:YAG & $\begin{array}{l}50 \mathrm{~mJ} / \mathrm{pulse}, \\
25 \mathrm{pulse} / \mathrm{s}\end{array}$ & $\begin{array}{l}24 \text { patients. Test } \\
\text { group: } 12 \text { patients; } \\
\text { Control group: } 12 \\
\text { patients }\end{array}$ & $\begin{array}{l}\text { Randomized } \\
\text { controlled clinical } \\
\text { trial. Control group: } \\
\text { surgical regenerative } \\
\text { therapy, involving } \\
\text { mechanical } \\
\text { debridement and } \\
\text { GBR. Test group: } \\
\text { adjunctive laser } \\
\text { irradiation in } \\
\text { addition to } \\
\text { mechanical } \\
\text { debridement prior to } \\
\text { bone grafting }\end{array}$ & 6 months & $\begin{array}{l}\text { PD reductions } \\
\text { was higher in test } \\
\text { group vs. control. } \\
\text { No statistical } \\
\text { differences were } \\
\text { found in CAL } \\
\text { gain }(1.90 \pm 2.28 \\
\text { vs. } 1.47 \pm 1.76 \\
\text { mm; test vs. } \\
\text { control), GI } \\
\text { reduction }(-1.14 \\
\pm 1.15 \text { vs. }-1.04 \\
\pm 0.89 ; \text { test vs. } \\
\text { control), } \\
\text { radiographic } \\
\text { linear bone gain } \\
(1.27 \pm 1.14 \text { vs. } \\
1.08 \pm 1.04 \text { mm; } \\
\text { test vs. control) } \\
\text { or proportional } \\
\text { defect size } \\
\text { reduction } \\
\text { (-24.46 } \pm 19.00 \% \\
\text { vs. }-15.19 \pm \\
23.56 \% ; \text { test vs. } \\
\text { control) }\end{array}$ & $\begin{array}{l}\text { The use of } \\
\text { Er:YAG laser was } \\
\text { effective in PPD } \\
\text { reduction }\end{array}$ \\
\hline
\end{tabular}

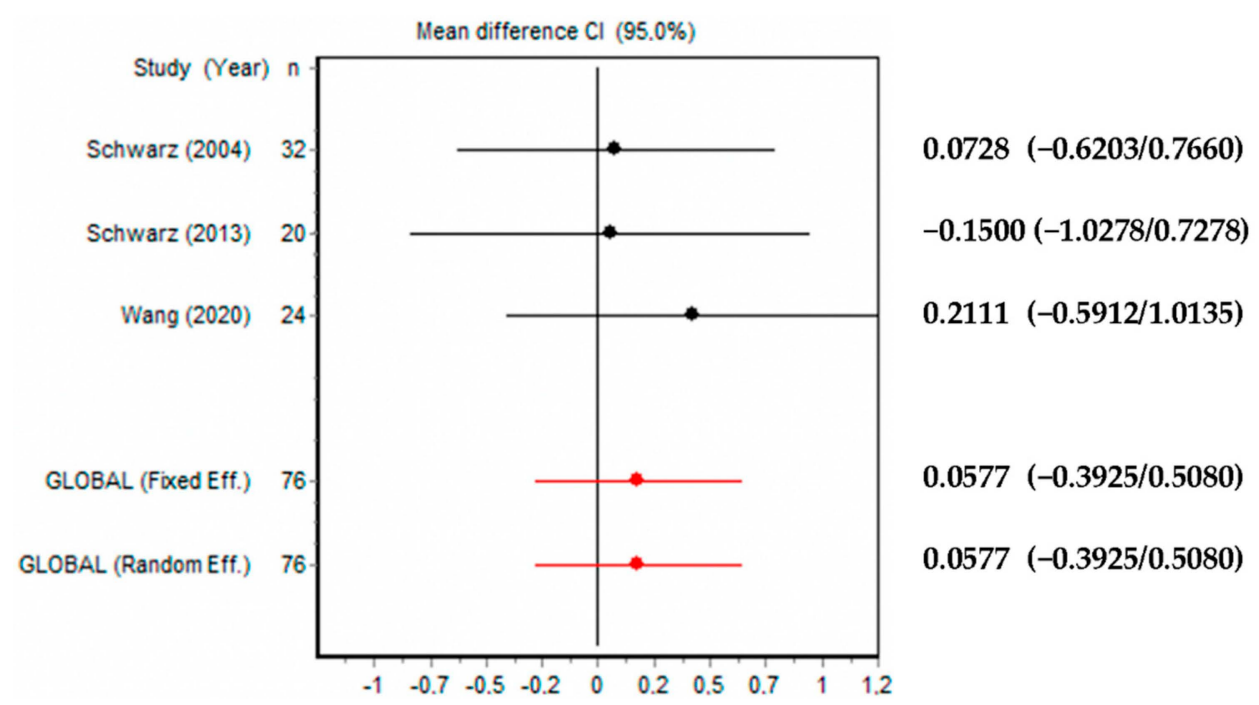

Figure 3. Forest Plot for PPD reduction mean after treatment (Er:YAG laser vs. conventional mechanical therapy). 
Table 2. Use of $\mathrm{CO}_{2}$ laser in peri-implantitis treatment.

\begin{tabular}{|c|c|c|c|c|c|c|c|}
\hline $\begin{array}{l}\text { First } \\
\text { Author- } \\
\text { Year }\end{array}$ & $\begin{array}{l}\text { Type } \\
\text { of } \\
\text { Laser }\end{array}$ & $\begin{array}{l}\text { Laser } \\
\text { Settings }\end{array}$ & $\begin{array}{l}\text { No. of } \\
\text { Patients }\end{array}$ & Study Design & $\begin{array}{l}\text { Follow-Up } \\
\text { Duration }\end{array}$ & $\begin{array}{l}\text { Results Obtained at the } \\
\text { End of the Follow-Up }\end{array}$ & Conclusions \\
\hline $\begin{array}{c}\text { Romanos } \\
\text { et al., 2008 } \\
{[17]}\end{array}$ & $\mathrm{CO}_{2}$ & $\begin{array}{c}\text { Wavelength } \\
10.6 \mathrm{~nm} \text { at } \\
7 \mathrm{~W}\end{array}$ & $\begin{array}{c}73 \\
\text { implants. } \\
\text { Group 1: } \\
19 \\
\text { implants; } \\
\text { Group 2: } \\
15 \\
\text { implants; } \\
\text { Group 3: } \\
22 \\
\text { implants; } \\
\text { Group 4: } \\
\text { 17 } \\
\text { implants }\end{array}$ & $\begin{array}{c}\text { Clinical Report. } \\
\text { Group 1: } \\
\text { Conventional } \\
\text { decontamination + } \\
\text { soft tissue resection. } \\
\text { Group 2: } \\
\text { Conventional } \\
\text { decontamination + } \\
\text { bone augmentation. } \\
\text { Group 3: Laser } \\
\text { decontamination + } \\
\text { soft tissue resection. } \\
\text { Group 4: Laser } \\
\text { decontamination }+ \\
\text { bone augmentation }\end{array}$ & $\begin{array}{c}\text { Mean } \\
\text { post-operative } \\
\text { observational } \\
\text { period } 27.10 \pm \\
17.83 \text { months }\end{array}$ & 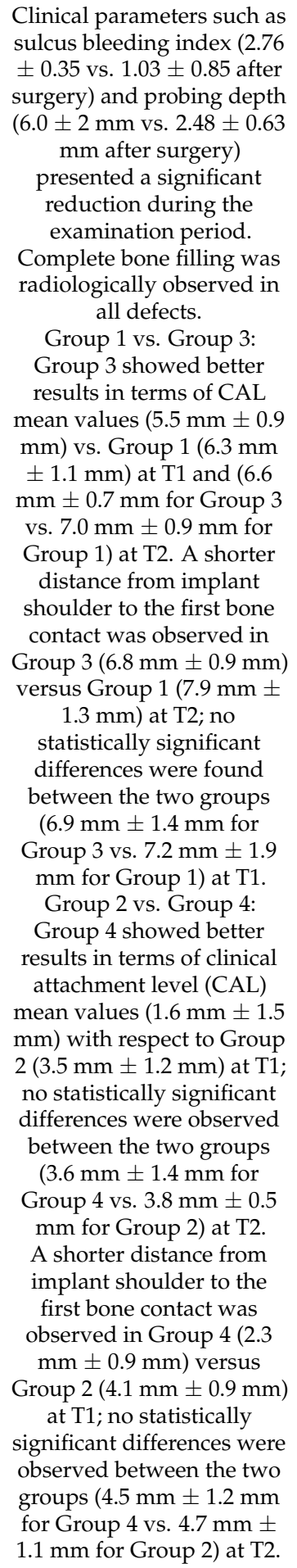 & $\begin{array}{l}\text { In this study, } \mathrm{CO}_{2} \\
\text { laser } \\
\text { decontamination } \\
\text { associated with } \\
\text { regenerative } \\
\text { surgery was } \\
\text { effective in the } \\
\text { treatment of } \\
\text { peri-implantitis. } \\
\\
\text { dissue resection. }\end{array}$ \\
\hline
\end{tabular}


Table 3. Use of diode laser in peri-implantitis treatment.

\begin{tabular}{|c|c|c|c|c|c|c|c|}
\hline $\begin{array}{l}\text { First } \\
\text { Author- } \\
\text { Year }\end{array}$ & $\begin{array}{l}\text { Type } \\
\text { of } \\
\text { Laser }\end{array}$ & $\begin{array}{l}\text { Laser } \\
\text { Settings }\end{array}$ & $\begin{array}{l}\text { No. of } \\
\text { Patients }\end{array}$ & Study Design & $\begin{array}{l}\text { Follow- } \\
\text { Up } \\
\text { Dura- } \\
\text { tion }\end{array}$ & $\begin{array}{l}\text { Results Obtained at the } \\
\text { End of the Follow-Up }\end{array}$ & Conclusions \\
\hline $\begin{array}{c}\text { Schar et al., } \\
2013 \\
\text { [19] }\end{array}$ & $\begin{array}{l}\text { Diode } \\
\text { laser + } \\
\text { dye } \\
\text { phe- } \\
\text { noth- } \\
\text { iazine } \\
\text { chlo- } \\
\text { ride }\end{array}$ & $\begin{array}{l}\text { Wavelength } \\
\text { of } 660 \mathrm{~nm} \text {; } \\
\text { Power } \\
\text { density of } \\
100 \mathrm{~mW}\end{array}$ & $\begin{array}{l}40 \text { patients. } \\
\text { Test group: } 20 \\
\text { patients } \\
\text { Control group: } \\
20 \text { patients }\end{array}$ & $\begin{array}{c}\text { Prospective } \\
\text { randomized clinical } \\
\text { trial. All implants } \\
\text { were mechanically } \\
\text { debrided with } \\
\text { titanium curettes and } \\
\text { with a glycine-based } \\
\text { powder air-polishing } \\
\text { system. Implants in } \\
\text { the test group } \\
\text { received adjunctive } \\
\text { photodynamic } \\
\text { therapy (PDT), } \\
\text { whereas minocycline } \\
\text { microspheres were } \\
\text { locally delivered into } \\
\text { the peri-implant } \\
\text { pockets of control } \\
\text { implants } \\
\text { Prospective }\end{array}$ & 6 months & $\begin{array}{l}\text { Complete resolution of } \\
\text { mucosal inflammation was } \\
\text { obtained in } 15 \% \text { of the } \\
\text { implants in the control } \\
\text { group and in } 30 \% \text { of the } \\
\text { implants in the test group } \\
\text { ( } p=0.16) \text {. Periodontal } \\
\text { probing depth (PPD), } \\
\text { mucosal recession, plaque } \\
\text { index (PI) and clinical } \\
\text { attachment level (CAL) } \\
\text { measurements did not yield } \\
\text { statistically significant } \\
\text { changes ( } p>0.05 \text { ) in both } \\
\text { groups }\end{array}$ & $\begin{array}{l}\text { Adjunctive PDT } \\
\text { may represent an } \\
\text { alternative } \\
\text { treatment modality } \\
\text { in the non-surgical } \\
\text { management of } \\
\text { initial } \\
\text { peri-implantitis }\end{array}$ \\
\hline $\begin{array}{l}\text { Bassetti } \\
\text { et al., 2014 } \\
\text { [20] }\end{array}$ & $\begin{array}{l}\text { Diode } \\
\text { laser + } \\
\text { dye } \\
\text { phe- } \\
\text { noth- } \\
\text { iazine } \\
\text { chlo- } \\
\text { ride }\end{array}$ & $\begin{array}{l}\text { Wavelength } \\
\text { of } 660 \mathrm{~nm} \text {; } \\
\text { Power } \\
\text { density of } \\
100 \mathrm{~mW}\end{array}$ & $\begin{array}{l}40 \text { patients. } \\
\text { Test group: } 20 \\
\text { patients } \\
\text { Control group: } \\
20 \text { patients }\end{array}$ & $\begin{array}{l}\text { Prospective } \\
\text { randomized clinical } \\
\text { trial. All implants } \\
\text { were mechanically } \\
\text { debrided with } \\
\text { titanium curettes and } \\
\text { with a glycine-based } \\
\text { powder air-polishing } \\
\text { system. Implants in } \\
\text { the test group } \\
\text { received adjunctive } \\
\text { PDT, whereas } \\
\text { minocycline } \\
\text { microspheres were } \\
\text { locally delivered into } \\
\text { the peri-implant } \\
\text { pockets of control } \\
\text { implants }\end{array}$ & $\begin{array}{c}12 \\
\text { months }\end{array}$ & $\begin{array}{l}\text { Complete resolution of } \\
\text { mucosal inflammation was } \\
\text { obtained in } 35 \% \text { of the } \\
\text { implants in the control } \\
\text { group and in } 41.1 \% \text { of the } \\
\text { implants in the test group } \\
(p=0.16) . \text { PPD, mucosal } \\
\text { recession, PI and CAL } \\
\text { measurements did not yield } \\
\text { statistically significant } \\
\text { changes }(p>0.05) \text { in both } \\
\text { groups }\end{array}$ & $\begin{array}{l}\text { Adjunctive PDT } \\
\text { may represent an } \\
\text { alternative } \\
\text { treatment modality } \\
\text { in the non-surgical } \\
\text { management of } \\
\text { initial } \\
\text { peri-implantitis }\end{array}$ \\
\hline $\begin{array}{c}\text { Deppe } \\
\text { et al., 2013 } \\
\text { [21] }\end{array}$ & $\begin{array}{l}\text { Diode } \\
\text { laser + } \\
\text { phe- } \\
\text { noth- } \\
\text { iazine } \\
\text { chlo- } \\
\text { ride }\end{array}$ & $\begin{array}{l}\text { Wavelength } \\
\text { of } 660 \mathrm{~nm} \text {; } \\
\text { Power } \\
\text { density of } \\
60 \mathrm{~mW}\end{array}$ & $\begin{array}{l}16 \text { patients } \\
\text { with a total of } \\
18 \text { dental } \\
\text { implant. } \\
\text { Group 1: } 10 \\
\text { implants. } \\
\text { Group 2: } 8 \\
\text { implants }\end{array}$ & $\begin{array}{l}\text { Clinical Pilot Study. } \\
\text { Group 1: }<5 \mathrm{~mm} \\
\text { bone loss around the } \\
\text { implant. Group } 2:>5 \\
\text { mm bone loss around } \\
\text { the implant. Each } \\
\text { group was treated by } \\
\text { photodynamic } \\
\text { therapy (PDT) }\end{array}$ & $\begin{array}{l}3 \text { months } \\
\text { and } 6 \\
\text { months }\end{array}$ & $\begin{array}{l}3 \text { months after therapy, in } \\
\text { both groups, sulcus } \\
\text { bleeding index and CAL } \\
\text { decreased significantly. In } \\
\text { contrast, after } 6 \text { months, } \\
\text { CAL and radiographic } \\
\text { evaluation of distance from } \\
\text { implant to bone (DIB) } \\
\text { increased significantly in } \\
\text { Group 2, not in Group } 1 . \\
\text { However, distance from } \\
\text { implant shoulder to } \\
\text { marginal mucosa were not } \\
\text { statistically different } 6 \\
\text { months after therapy in } \\
\text { both groups }\end{array}$ & $\begin{array}{l}\text { Nonsurgical PDT } \\
\text { could stop bone } \\
\text { resorption in } \\
\text { moderate } \\
\text { peri-implant } \\
\text { defects. }(<5 \mathrm{~mm}) \\
\text { but not in severe } \\
\text { defects }(>5 \mathrm{~mm})\end{array}$ \\
\hline
\end{tabular}


Table 3. Cont.

\begin{tabular}{|c|c|c|c|c|c|c|c|}
\hline $\begin{array}{l}\text { First } \\
\text { Author- } \\
\text { Year }\end{array}$ & $\begin{array}{l}\text { Type } \\
\text { of } \\
\text { Laser }\end{array}$ & $\begin{array}{l}\text { Laser } \\
\text { Settings }\end{array}$ & $\begin{array}{l}\text { No. of } \\
\text { Patients }\end{array}$ & Study Design & $\begin{array}{l}\text { Follow- } \\
\text { Up } \\
\text { Dura- } \\
\text { tion }\end{array}$ & $\begin{array}{l}\text { Results Obtained at the } \\
\text { End of the Follow-Up }\end{array}$ & Conclusions \\
\hline $\begin{array}{l}\text { Bombeccari } \\
\text { et al., } 2013 \\
\text { [22] }\end{array}$ & $\begin{array}{l}\text { Diode } \\
\text { laser }\end{array}$ & $\begin{array}{l}\text { Wavelength } \\
\text { of } 810 \mathrm{~nm} \\
\text { and a con- } \\
\text { tinuous } \\
\text { wave } \\
\text { mode of } 1 \\
\text { W }\end{array}$ & $\begin{array}{l}40 \text { patients. } \\
\text { Test group: } 20 \\
\text { patients. } \\
\text { Control group: } \\
20 \text { patients }\end{array}$ & $\begin{array}{l}\text { Randomized } \\
\text { comparative } \\
\text { case-control study. } \\
\text { The aim of this study } \\
\text { was to compare the } \\
\text { efficacy of } \\
\text { antimicrobial PDT in } \\
\text { the test group ( } \mathrm{N}= \\
20 \text { ) versus } \\
\text { conventionally } \\
\text { surgical therapy in } \\
\text { the control group (N } \\
=20 \text { ), in patients with } \\
\text { peri-implantitis }\end{array}$ & 6 months & $\begin{array}{l}\text { Total anaerobic counts of } \\
\text { bacteria did not differ } \\
\text { significantly between the } \\
\text { two groups (test group, } \\
\text { 95.2\%; control group } \\
80.85 \% \text { ). Bleeding on } \\
\text { probing (BOP) at } 6 \text { months } \\
\text { after treatment was } \\
\text { observed in adult } \\
\text { periodontally compromised } \\
\text { patients in both groups. } \\
\text { Changes in PPD were } \\
\text { recorded in the PDT group } \\
\text { only }\end{array}$ & $\begin{array}{l}\text { Treatment with } \\
\text { PDT in patients } \\
\text { with } \\
\text { peri-implantitis } \\
\text { was not associated } \\
\text { with major } \\
\text { reduction of total } \\
\text { anaerobic bacteria } \\
\text { on the rough } \\
\text { surfaces of dental } \\
\text { implants as } \\
\text { compared with } \\
\text { surgical therapy }\end{array}$ \\
\hline $\begin{array}{l}\text { Papadopoulos } \\
\text { et al., 2015 } \\
\text { [23] }\end{array}$ & $\begin{array}{l}\text { Diode } \\
\text { laser }\end{array}$ & $\begin{array}{l}\text { Wavelength } \\
\text { of } 980 \mathrm{~nm} \\
0.8 \mathrm{~W} \text { in } \\
\text { pulsed } \\
\text { mode }\end{array}$ & $\begin{array}{c}19 \text { patients. } \\
\text { Test group: } 9 . \\
\text { Control group: } \\
10\end{array}$ & $\begin{array}{l}\text { Randomized } \\
\text { comparative } \\
\text { case-control study. } \\
\text { Control group: } \\
\text { mucoperiosteal flaps, } \\
\text { implant surface } \\
\text { debridement with } \\
\text { plastic curettes and } \\
\text { sterilized gauzes } \\
\text { soaked in saline. Test } \\
\text { group: was treated } \\
\text { similarly but with } \\
\text { additional irradiation } \\
\text { using a diode laser } \\
\text { Randomized }\end{array}$ & 6 months & $\begin{array}{l}3 \text { months after treatment, a } \\
\text { mean PPD reduction of } 1.19 \\
\text { mm for the control group } \\
\text { and } 1.38 \mathrm{~mm} \text { for the test } \\
\text { group was recorded. The } \\
\text { corresponding BOP } \\
\text { changes were } 72.9 \text { and } \\
66.7 \% \text {, respectively. These } \\
\text { changes were significant } \\
\text { and remained at the same } \\
\text { levels at the } 6 \text {-month } \\
\text { examination }(p<0.05) .\end{array}$ & $\begin{array}{c}\text { Surgical treatment } \\
\text { of peri-implantitis } \\
\text { by access flaps } \\
\text { leads to } \\
\text { improvement of all } \\
\text { clinical parameters } \\
\text { studied while the } \\
\text { additional use of } \\
\text { diode laser does } \\
\text { not seem to have } \\
\text { an extra beneficiary } \\
\text { effect }\end{array}$ \\
\hline $\begin{array}{c}\text { Tenore } \\
\text { et al., 2020 } \\
\text { [24] }\end{array}$ & $\begin{array}{l}\text { Diode } \\
\text { laser }\end{array}$ & $\begin{array}{c}\text { Wavelength } \\
980 \mathrm{~nm} \text {, at } \\
\text { power of } \\
1 \mathrm{~W} \text { in } \\
\text { pulsed } \\
\text { mode }\end{array}$ & $\begin{array}{l}23 \text { patients } \\
\text { with a total of } \\
23 \text { dental } \\
\text { implants. Test } \\
\text { group: } 11 \\
\text { implants. } \\
\text { Control group: } \\
12 \text { implants }\end{array}$ & $\begin{array}{l}\text { Controlled Trial. For } \\
\text { both groups, full } \\
\text { mouth mechanical } \\
\text { debridement was per- } \\
\text { formed through the } \\
\text { use of ultrasonic } \\
\text { piezoelectric scaler } \\
\text { and manual } \\
\text { instruments. In test } \\
\text { group the } \\
\text { peri-implant sulcus } \\
\text { of each implant was } \\
\text { irradiated } \\
\text { immediately after the } \\
\text { mechanical } \\
\text { debridement with } \\
\text { laser }\end{array}$ & 3 months & $\begin{array}{c}\text { The average of PPD value } \\
\text { for test group was } 4.04 \pm \\
0.54 \mathrm{~mm} \text { at baseline and it } \\
\text { was } 2.98 \pm 0.7 \mathrm{~mm} \text { after } 3 \\
\text { months. In the control } \\
\text { group, the average was } 3.8 \\
\pm 1.24 \mathrm{~mm} \text { at baseline and } \\
\text { was } 3.54 \pm 0.35 \mathrm{~mm} \text { after } 3 \\
\text { months. A greater decrease } \\
\text { in both clinical parameters } \\
\text { was detected for subjects } \\
\text { treated with lasers (test } \\
\text { group) }\end{array}$ & $\begin{array}{l}\text { Diode laser may be } \\
\text { considered an } \\
\text { adjunct to the } \\
\text { conventional } \\
\text { non-surgical } \\
\text { treatments of } \\
\text { peri-implants } \\
\text { mucositis and } \\
\text { initial } \\
\text { peri-implantitis }\end{array}$ \\
\hline $\begin{array}{c}\text { Mettraux } \\
\text { et al., 2016 } \\
\text { [25] }\end{array}$ & $\begin{array}{l}\text { Diode } \\
\text { laser }\end{array}$ & $\begin{array}{c}\text { Wavelength } \\
810 \mathrm{~nm}, 2.5 \\
\text { W, } 50 \mathrm{~Hz} \\
10 \mathrm{~ms}\end{array}$ & $\begin{array}{l}15 \text { patients } \\
\text { with a total of } \\
23 \text { dental } \\
\text { implants. }\end{array}$ & $\begin{array}{l}\text { Retrospective case } \\
\text { series study. The } \\
\text { implant surfaces } \\
\text { were debrided under } \\
\text { local anesthesia using } \\
\text { carbon fiber curettes, } \\
\text { and the inflamed } \\
\text { peri-implant soft } \\
\text { tissue wall was } \\
\text { curetted with sharp } \\
\text { metal curettes. } \\
\text { Adjunctive diode } \\
\text { laser was applied }\end{array}$ & 2 years & $\begin{array}{c}\text { The deepest PPD decreased } \\
\text { from } 7.5 \pm 2.6 \mathrm{~mm} \text { to } 3.6 \pm \\
0.7 \mathrm{~mm} \text { at buccal }(p< \\
0.0001) \text { and from } 7.7 \pm 2.1 \\
\text { mm to } 3.8 \pm 0.9 \mathrm{~mm} \text { at oral } \\
\text { sites }(p<0.0001) \text {, } \\
\text { respectively. The } \% \text { of } \\
\text { implants with } \geq 1 \text { site with } \\
\text { BoP decreased from } 100 \% \\
\text { at BL to } 43 \% \text { after } 2 \text { years ( } p \\
=0.0002) \text {. The } \% \text { of } \\
\text { implants with suppuration } \\
\text { decreased from } 87 \% \text { at BL } \\
\text { to } 0 \% \text { after } 2 \text { years }(p< \\
0.0001)\end{array}$ & $\begin{array}{l}\text { Non-surgical } \\
\text { mechanical therapy } \\
\text { of PI with } \\
\text { adjunctive } \\
\text { repeated } \\
\text { application of a } \\
\text { diode laser yielded } \\
\text { significant clinical } \\
\text { improvements } \\
\text { after an } \\
\text { observation period } \\
\text { of at least } 2 \text { years }\end{array}$ \\
\hline
\end{tabular}




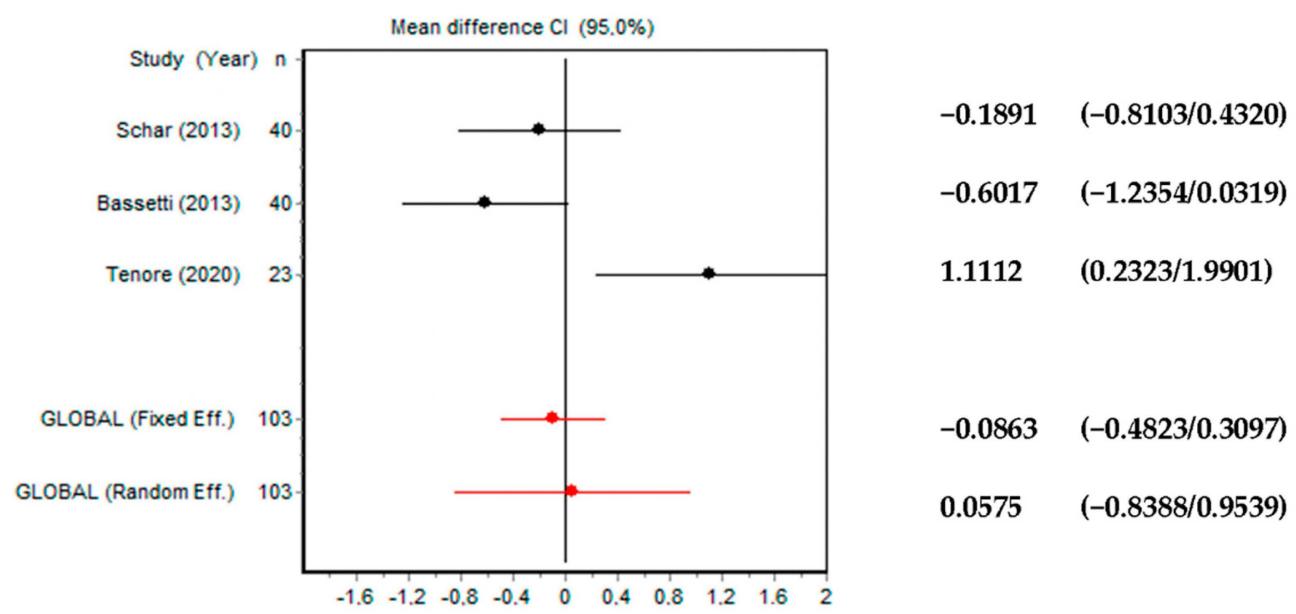

Figure 4. Forest plot for PPD reduction mean (diode laser vs. conventional mechanical therapy).

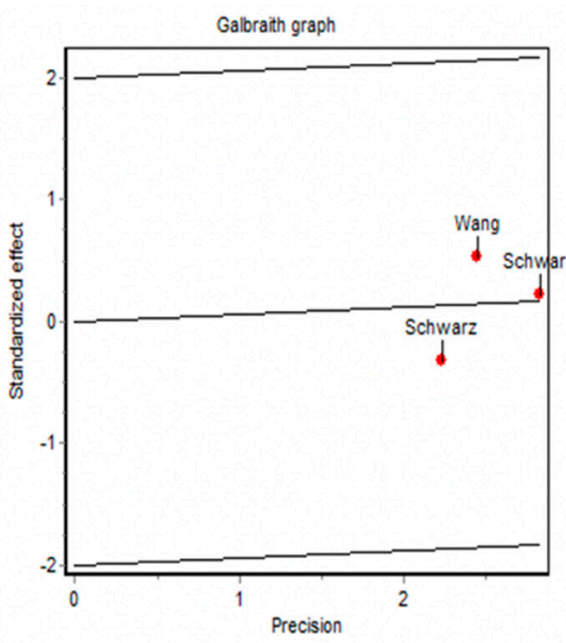

a)

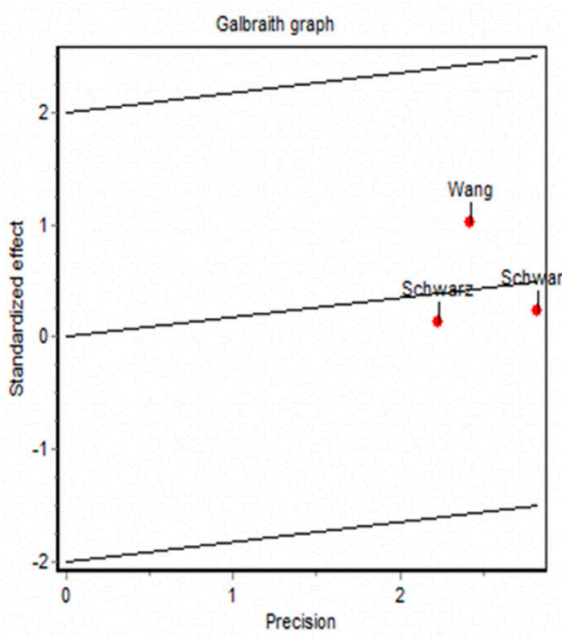

b)

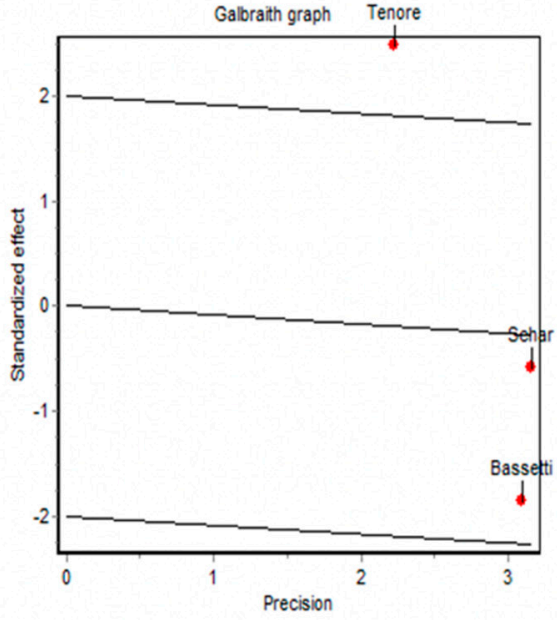

c)

Figure 5. Galbraith graph to show the heterogeneity of the analyzed study: (a) CAL gain in Er:YAG laser group ( $p=0.7854)$, (b) PPD reduction in Er:YAG laser group $(p=0.8364)$ and (c) PPD reduction in diode laser group $(p=0.0075)$.

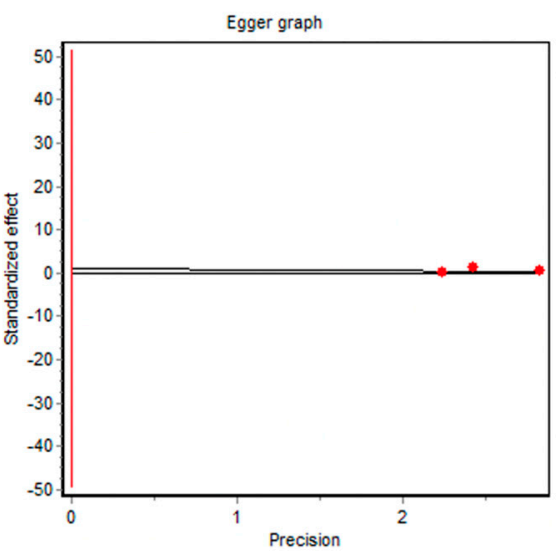

a)

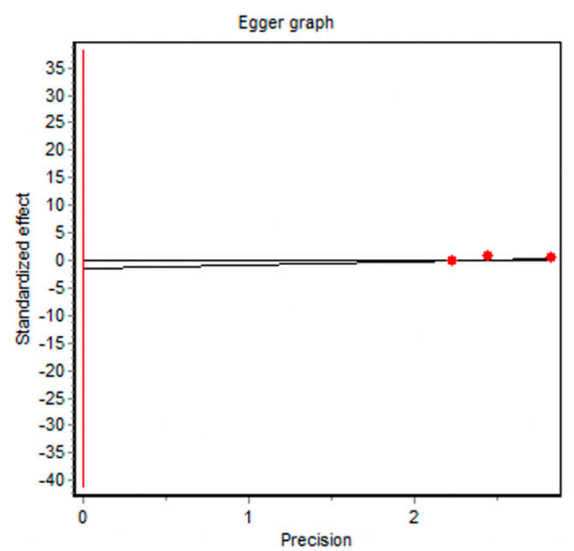

b)

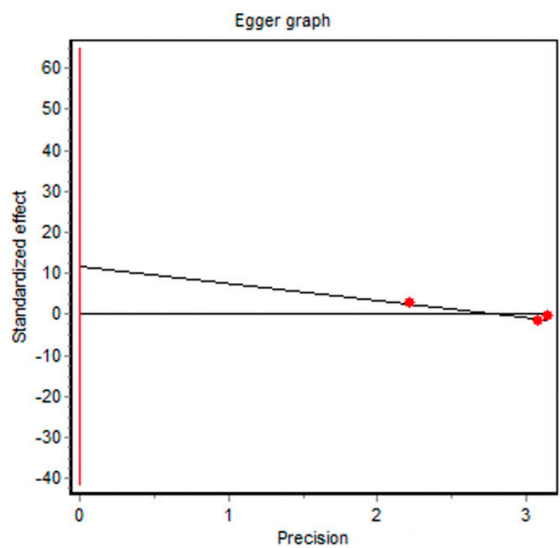

c)

Figure 6. Egger graph to show the risk of bias in the analyzed study: (a) CAL gain in Er:YAG laser group $(p=0.82),(\mathbf{b})$ PPD reduction in Er:YAG laser group $(p=0.69)$ and $(c)$ PPD reduction in diode laser group $(p=0.22)$. 


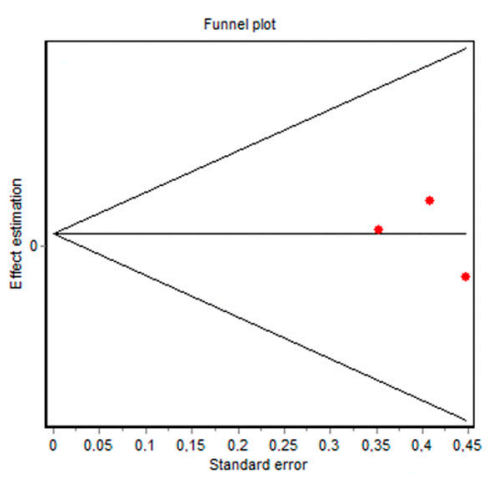

a)

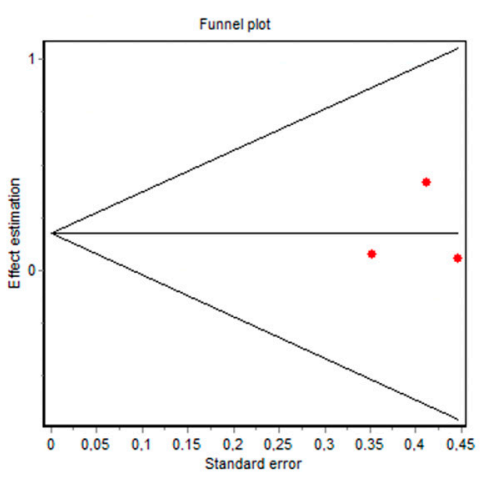

b)

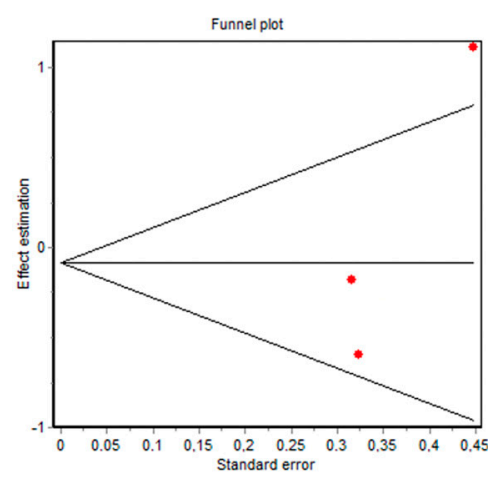

c)

Figure 7. Funnel plot to show the risk of bias in the analyzed study: (a) CAL gain in Er:YAG laser group ( $p=1.0000)$, (b) PPD reduction in Er:YAG laser group $(p=1.0000)$ and (c) PPD reduction in diode laser group $(p=1.0000)$.

\subsection{Group 1-Use of Er:YAG Laser in Peri-Implantitis Treatment (Table 1)}

Schwarz et al. tested the effectiveness of an Er:YAG laser with mechanical debridement in a randomized control trial [12]. Twenty patients with a total of 32 dental implants affected by moderate-to-severe peri-implant lesions were treated by using a Er: YAG laser with a cone-shaped glass-fiber tip with a wavelength of $2.9 \mathrm{~nm}$ and a power of $100 \mathrm{~mJ} /$ pulse (test group), or mechanical debridement with plastic curette and antiseptic treatment with $0.2 \%$ chlorhexidine digluconate (control group). Plaque index (PI), bleeding on probing $(\mathrm{BOP})$, probing depth (PD), gingival recession (GR) and clinical attachment level (CAL) were measured at the baseline and after 3 and 6 months. After 6 months, the BOP mean value decreased in the test group from the initial $83 \%$ to $31 \%(p<0.001)$, and in the control group, it decreased from $80 \%$ to $58 \%(p<0.001)$. The sites treated with the Er:YAG laser showed a mean change in CAL from $5.8 \pm 1 \mathrm{~mm}$ (baseline) to $5.1 \pm 1.1 \mathrm{~mm}(p<0.01)$ after 6 months. In the control group, patients showed a mean change in CAL from $6.2 \pm 1.5 \mathrm{~mm}$ (baseline) to $5.6 \pm 1.6 \mathrm{~mm}(p<0.001)$ after 6 months; consequently, the difference between the two groups was not statistically significant $(p<0.05)$ [12].

Similar results were found by Renvert after conducting a randomized controlled trial on 42 patients equally distributed in a control and a test group with the purpose to compare abrasive air-flow therapy versus Er:YAG laser therapy [13]. No statistically different results were achieved between the control and test group after 6 months, in terms of bleeding on probing and probing depth.

Another study conducted by Schwarz et al. revealed no statistically significant differences between the application of an Er:YAG (ERL) and conventional plastic curette + sterile saline therapy (CPS) [14]. In this work, the authors evaluated the clinical outcomes obtained after a 48-month follow-up between a test group treated with ERL and a control group treated with CPS. Patients of both groups were preliminarily subjected to a complete surgical granulation tissue remotion and implantoplasty in the exposed fixtures parts. Unmodified implant surface areas were subsequently randomly treated with Er:YAG laser instrumentation (test group) or with plastic curettes and cotton pellets soaked in sterile saline (control group). Finally, in both groups, intrabony defects were treated with bovine bone (NBM) and coated with a collagen membrane (CM). Clinically significant improvements in BOP values and significant gains in PD and CAL were observed in both CPS and ERL groups. After 24 months, in ERL was found a greater reduction in BOP if compared with CPS group (ERL: $75.0 \pm 32.6 \%$ vs. CPS: $54.9 \pm 30.3 \%$ ). However, after 48 months, the CPS-treated group was associated with a greater reduction in BOP, while the ERL-treated sites appeared to have relapsed.

A comparative study conducted in Austria by Pommer et al. analyzed the results obtained with three different therapeutic interventions for peri-implantitis treatments [15] Seventy-two patients were treated by Er:YAG laser decontamination (Group 1), 47 with implantoplasty (Group 2) and 23 with a combination of both techniques (Group 3). The 
overall treatment success rate was $88.7 \%$ after 9 years of follow-up. The success rate following laser decontamination, implantoplasty and a combination of both therapeutic approaches was $91.9 \%, 87.2 \%$ and $82.6 \%$, respectively. Implant failures were found after $4.9 \pm 1.9$ years of treatment (on average), with no substantial differences between the three groups $(p=0.303)$ : six implant failures $(8 \%)$ after laser decontamination, six implant failures (13\%) after implantoplasty and four implant failures (17\%) after combining the two therapies. The results founded by the authors suggest that success rates of peri-implantitis therapy did not differ between laser decontamination and/or implantoplasty surgery.

Wang proposed the use of Er:YAG laser to assist surgical regenerative peri-implantitis therapy in the presence of bone defects [16]. Twenty-four patients with infrabony defects caused by peri-implant disease were randomly divided into two groups (test group and control group). All patients underwent surgical therapy with implantoplasty and bone graft covered with dermal matrix membrane. In addition, in the test group was performed Er:YAG laser irradiation processes, such as adjunctive therapy, to remove inflammatory tissue and to decontaminate the implant surface from the presence of pathogens. Six months after surgical therapy, patients of both groups showed improvements in PD, gingival index (GI) and CAL. Between the two groups, no statistical differences were found in CAL gain (test group, $1.90 \pm 2.28$; control group, $1.47 \pm 1.76 \mathrm{~mm}$ ), GI reduction (test group, $-1.14 \pm 1.15$; control group, $-1.04 \pm 0.89$ ), radiographic linear bone gain (test group, $1.27 \pm 1.14$; control group, $1.08 \pm 1.04 \mathrm{~mm}$ ) or proportional defect size reduction (test group, $-24.46 \pm 19.00 \%$; control group, $-15.19 \pm 23.56 \%$ ). Instead, a higher PD reduction was found in the test compared to the control group (test group, $2.65 \pm 2.14$; control group, $1.85 \pm 1.71 \mathrm{~mm} ; p=0.014)$.

\subsection{Group 2-Use of $\mathrm{CO}_{2}$ Laser in Peri-Implantitis Treatment (Table 2)}

Romanos presented a case series focused on the treatment of the peri-implant infrabony defects with $\mathrm{CO}_{2}$ laser irradiation [17]. After a full-thickness mucoperiosteal flap elevation and the remotion of granulomatous tissue to obtain the implant exposure and the access to peri-implant infrabony defect, $\mathrm{CO}_{2}$ laser irradiation was performed to achieve the implant surface decontamination and to promote blood coagulation in the bone defect. Subsequently guided bone regeneration technique was applied to achieve a complete filling of the infrabony defects. After an observation period of $27.10 \pm 17.83$ months, no periimplant inflammatory was noted; clinical parameters such as sulcus bleeding index and probing depth presented a statistically significant reduction during the examination period $(p<0.01)$. In addition, a complete bone filling was radiologically observed in all defects.

Deppe and his co-worker conducted a study with the purpose to determine whether $\mathrm{CO}_{2}$-laser-assisted decontamination is effective in the treatment of ailing implants in humans [18]. Thirty-two patients with 73 ailing implants were considered in this evaluation and subsequently included in four groups on the basis of treatment to be performed (Group 1, conventional decontamination + soft tissue resection; Group 2, conventional decontamination + bone augmentation; Group 3, laser decontamination + soft tissue resection; Group 4, laser decontamination + bone augmentation. The outcomes of the four different treatments were evaluated and compared (Group 2 vs. Group 4; Group 1 vs. Group 3) 4 and 59 months after surgery. Following the results obtained from the present study, laser-assisted implant decontamination + soft tissue resection (Group 3) resulted in significantly more favorable levels of clinical attachment than conventional decontamination + soft tissue resection (Group 1) at 4, as well as 59, months after surgery. A shorter distance from implant shoulder to the first bone contact was observed in Group $3(6.8 \mathrm{~mm} \pm 0.9 \mathrm{~mm})$ versus Group 1 (7.9 $\mathrm{mm} \pm 1.3 \mathrm{~mm}) 59$ months after surgery; no statistically significant differences were found between the two groups $(6.9 \mathrm{~mm} \pm 1.4 \mathrm{~mm}$ for Group 3 vs. $7.2 \mathrm{~mm} \pm 1.9 \mathrm{~mm}$ for Group 1) after 4 months from surgery. A similar result was also found in the groups where bone augmentation was performed: Group 4 showed better results in terms of clinical attachment level (CAL) mean values $(1.6 \mathrm{~mm} \pm 1.5 \mathrm{~mm})$ with respect to Group $2(3.5 \mathrm{~mm} \pm 1.2 \mathrm{~mm})$, and a shorter distance from implant shoulder 
to the first bone contact was observed in Group $4(2.3 \mathrm{~mm} \pm 0.9 \mathrm{~mm})$ versus Group 2 $(4.1 \mathrm{~mm} \pm 0.9 \mathrm{~mm}) 4$ months after surgery. The authors noted that the use of $\mathrm{CO}_{2}$ laser concomitant with soft tissue resection was effective in the treatment of peri-implantitis.

\subsection{Group 3-Use of Diode Laser in Peri-Implantitis Treatment (Table 3)}

Photodynamic therapy (PDT) with diode laser in the non-surgical therapy of periimplantitis was studied by Schär [19]. Forty subjects with 4-6 mm PPD, bleeding on probing and marginal bone loss were randomly divided into two groups, according to the administered treatment. Patients in the control group received exclusively local drugs delivery (LLD) in the peri-implants pocket. After 3 months of healing, implants showed a significant BOP reduction $(p<0.0001)$ compared to the initial situation in both groups. After 6 months, complete resolution of the mucosal inflammation was found in $30 \%$ of cases treated with PDT and 15\% in the LDD group. The authors conclude their analysis by stating that the adjunctive PDT may represent a reliable treatment in the non-surgical therapy of peri-implantitis.

Bassetti et al. presented the outcomes of the same study but with a follow-up of 12 months. The authors observed a statistically significant $(p<0.05)$ decrease in PPD from baseline after conventional treatment followed by laser irradiation [20]. In accordance with the results of this study, mechanical treatment accompanied by photodynamic therapy could favor an improvement of clinical parameters in cases of mild or moderate peri-implantitis.

Positive effects of the diode laser in the PDT mode on the treatment of PI with PPD $<5 \mathrm{~mm}$ were highlighted also by Deppe [21]. The evaluation was conducted in patients with mild (PPD less than $5 \mathrm{~mm}$ ) and severe (PPD more than $5 \mathrm{~mm}$ ) PI. In all patients, a reduction of BOP and PPD was observed 3 months after conventional nonsurgical therapy followed by diode laser irradiation. However, in the patients affected by severe PI, BOP reduction was not maintained, and a slight increase in PPD was observed after 6 months.

Bombeccari et al. divided 20 patients in four groups: two test groups treated with photodynamic therapy + conventional manual therapy (PDT $+\mathrm{CT}$ ) and two control groups treated only with conventional therapy (CT) [22]. Patients were also divided into two groups according to bone defects' depth ( $\leq 2 \mathrm{~mm}$ or $\geq 2 \mathrm{~mm}$ ). In defects more than $2 \mathrm{~mm}$, a mucoperiosteal flap was carried out. CT consisted in scaling associated to chlorhexidine irrigation; PDT consisted in applying blue into peri-implantitis site and irradiating it with a diode laser for $1.5 \mathrm{~min}$ in a continuous wave mode. Immediately after treatment, bacterial-count results showed a 93.6\% reduction in the PDT + TC group and 83.5\% in the control group. However, a recolonization of some bacterial species emerged at 6 months. The authors concluded that PDT can be effectively used as a supplement to conventional therapy.

Papadopoulus tested the effectiveness of the adjunctive diode laser irradiation during the surgical treatment of peri-implantitis [23]. Nineteen patients were enrolled in this clinical trial, and, on all of them, surgical regenerative therapy of the peri-implant infrabony defects was performed. Only for 10 randomly chosen patients (test group) was an additional irradiation with diode laser performed, with the aim to improve the decontamination of the infected implant parts and of the surrounding tissues.

Clinical peri-implant indices (CAL, PPD, BOP and PI) were recorded into clinical chart at the baseline and at 3 and 6 months after treatment. Patients who received additional laser therapy showed a better CAL gain, but no substantial differences were found regarding the efficiency in the reduction of the other analyzed indices at 3 and 6 months after surgery $(p<0.05)$. Therefore, following the results obtained in this study, the additional use of the diode laser does not seem to have given supplementary clinical advantages in surgical management of peri-implantitis [23].

Tenore et al. treated 23 patients affected by mucositis (PiM) and initial peri-implantitis (Pi) [24]. The patients enrolled in the study were assigned to one of two groups: the 
control group (CG) was treated with conventional surgical therapy; and the test group (TG) was treated with a combination of conventional non-surgical therapy, with the addition decontamination with diode laser $(980 \mathrm{~nm})$. The clinical outcomes obtained were analyzed 3 months after treatment and showed greater improvements in BOP and PPD reduction in TG if compared with CG [24]. In particular, the mean reduction of PPD for TG was $4.04 \pm 0.54 \mathrm{~mm}$ at baseline and $2.98 \pm 0.70 \mathrm{~mm}$; in the CG, it was $3.8 \pm 1.24 \mathrm{~mm}$ at baseline and $3.54 \pm 0.35 \mathrm{~mm}$ after 3 months. BOP index was positive in 44 sites at baseline and only in six sites 3 months after laser treatment. In the CG, BOP reduction was less significative, going from 52 sites at baseline to 28 sites 3 months after conventional non-surgical therapy. The authors concluded that a $980 \mathrm{~nm}$ diode laser may be effective in non-surgical treatments of PiM and initial PI.

The effectiveness of adjunctive laser irradiation in non-surgical peri-implant therapy was highlighted in the retrospective analysis conducted by Mettraux [25]. The authors treated 23 infected implant surfaces with PPD $>5 \mathrm{~mm}$ with conventional mechanical debridement and by rinsing with sterile saline solution. Subsequently, adjunctive diode laser irradiation was performed in the pocket around the implant. All the described protocols were performed at the baseline and repeated after 7 and 14 days. After an observational period of 2 years, the authors noted an improvement of peri-implant health, with a significant reduction in all analyzed peri-implant indices (CAL, PPD, BOP and sulcular suppuration).

\section{Discussion}

Peri-implantitis treatment is still a challenge for the clinicians. To be successful, it is mandatory to perform a deep decontamination of the infected tissues around the dental implants, as well as the exposed fixture parts.

With the aim to improve the implant osseointegration, in the last years, scientists have developed anodized, machinated and sandblasted dental implant surfaces, which, due to their high roughness, are difficult to decontaminate; moreover, an optimal treatment procedure or a suitable instrument is not yet available.

In the literature, several approaches (mechanical-manual decontamination techniques, disinfection using chemical agents, implantoplasty of exposed fixture parts, laser therapy) were proposed for the decontamination of the infected implant parts by different authors, but there is still no consensus on which is the most predictable, reproducible and effective procedure [26].

Because of their ablation, bactericidal and bio-stimulator effects, dental lasers were proposed, including a utility tool for decontaminating the infected implant surfaces. However not all of them proved effective, and their improper use or settings could cause damage to peri-implant tissue and/or to implant fixtures. The main problem related to dental-laser use is the thermal effect on the titanium implant surfaces; in fact, its overheating can cause an increase of bone temperature over $47^{\circ} \mathrm{C}$ with heat-induced bone injury and compromise osseointegration. Another key point is the possibility that the radiation emitted by the dental laser, because of its moderate absorption rate on titanium, could damage the implant fixtures, for example, with crater formation on their surface. However, these events are rarely reported in the in vivo study and mainly depend on the improper choice of the dental laser type and on the irradiation parameters settings. Er:YAG, $\mathrm{CO}_{2}$ and diode lasers are the most documented due to their efficacy and more reliable application; when used with the correct emission power and for the appropriate time, it is scientifically known that they do not generate alterations of the implant surfaces and of the bone that surrounds them [11].

Giannelli published a paper to show the results obtained after testing the possible presence of thermal damage induced by $\lambda=808 \mathrm{~nm}$ GaAlAs diode laser irradiation on various titanium discs [27]. The aim of the authors was to identify, in vitro, the optimal laser settings to decontaminate the different implant surfaces with a diode laser $(\lambda=808 \mathrm{~nm})$. The outcome of these experiments showed a different absorption of laser radiation and, 
therefore, a different overheating depending on the manufacturing characteristics of the titanium surfaces. In particular, the highest temperature values were obtained on the rough fixture parts, corresponding to the endo-osseous implant portion. In contrast, the glossy implant parts caused lesser heating and faster cooling because of the high reflectivity of the surfaces. Laser radiation scattered from the glossy implant surface can induce undesired damage on the peri-implant and surrounding tissues. However, by using appropriate setting parameters, the laser radiation can be absorbed by adjacent periodontal tissues, promoting bio-stimulation and anti-phlogistic effect. The authors concluded their analysis by specifying that appropriate cautions must be dedicated when treating the implants parts in contact with alveolar bone to avoid overheating-related injuries, and irradiation parameters should be tailored.

Kreisler and his co-workers analyzed the effects of Neodymium-Doped Yttrium Aluminum Garnet (Nd:YAG) and Holmium-Doped Yttrium Aluminum Garnet (Ho:YAG), Er:YAG, $\mathrm{CO}_{2}$ and GaAlAs (diode laser) lasers with an electron microscope on four types of implant surfaces: mechanized, with sandblasting and treatment acid, titanium plasma sprayed and hydroxyapatite-coated [28]. The results obtained, in contrast to the findings of Giannelli's work, showed that diode laser was considered the safest for application on any type of surface, because it does not damage the titanium. Nd:YAG and Ho:YAG lasers determined the complete elimination of bacterial biofilm on titanium surfaces; however, the irradiation at standard energy emission settings could cause significant damage on the titanium surfaces of the fixture, making them unsuitable for decontamination of implant independently of the power output. $\mathrm{CO}_{2}$ and Er:YAG lasers, with the appropriate settings and wavelengths, could be used without damaging the implant surface and bone [28].

Following the findings reported by the paper published by Ganz, the $\mathrm{CO}_{2}$ laser did not cause a temperature change in excess of $3.9^{\circ} \mathrm{C}$ if used with a power density of 2 and $4 \mathrm{~W}$ in a continuous mode [29].

Er:YAG laser irradiation at high energy levels produces over-heating and minor damage on titanium-implant surfaces, Matsuyama showed that the use of cooling sources, such as water spray, drastically reduce the temperature elevation of the implant during the laser irradiation [30]. Taniguchi et al. reported that Er:YAG irradiation at pulse energy with water spray in near-contact mode caused no damage and was effective for debriding microstructured implant surfaces [31].

Following the clinical studies included in our paper, Er:YAG is the best laser system to decontaminate the infected implant parts and infrabony defects. The radiation emitted by the Er:YAG laser is highly absorbed by water molecules contained within hard and soft tissue of the oral cavity. Their consequent evaporation involves the production of micro-explosion and macro-molecular fragmentation in the target tissue without significative thermal variation. This phenomenon is the rationale of the ablative effects of this laser especially on the hard tissues [32]. For this reason, Er:YAG lasers, unlike diode and $\mathrm{CO}_{2}$ lasers, can be used in bone surgery and in the remotion of subgingival calculus in periodontal pockets. Some authors have compared the effectiveness of Er:YAG lasers' irradiation with conventional subgingival mechanical instrumentation (plastic curettes and air-polishing) in non-surgical peri-implant therapy [14-16]. Following the studies included in our review, it emerged that the Er:YAG laser was effective to complete the ablation of any residual subgingival bacteria from implant surfaces, and the difference in terms of therapeutic efficacy was not clinically significant when compared with conventional mechanical therapy. However, on the basis of the same clinical result obtained, conventional manual instrumentation requires manual skills and more time spent than laser irradiation. In surgical-regenerative therapy of peri-implant defects, Er.YAG lasers cannot replace conventional instrumentation and can be used like adjunctive therapy to remove inflammatory tissues and to complete the decontamination of the infected implants parts and surrounding hard and soft tissues [16].

Although the Er:YAG laser seems to be the most effective in the therapy of periimplantitis, the diode laser is the most tested by the authors. Its greater diffusion is 
probably related to its simplicity of use, its versatility and its cost-effectiveness compared to other laser systems. Diode is a deeply penetrating laser-type and shows the best cutting, coagulation and biostimulation activity in oral surgery [32]. In the treatment of periimplantitis, photodynamic therapy combined with traditional non-surgical therapy seems to have positive effects in the treatment of mucositis or peri-implantitis, in particular that with PPD less than $5 \mathrm{~mm}$ [21]. This result could be explained as the consequence of the antibacterial and bio-stimulating effects induced by the laser light on the periodontal tissues and on the exposed implant surfaces. Consequently, diode lasers, similar to $\mathrm{CO}_{2}$ lasers, have proved to be useful in obtaining a better disinfection of the implant surfaces colonized by bacteria and in stimulating the epithelial healing processes [18].

The clinical trials included in our paper highlighted that there is no single method for decontamination of implant surfaces, which is the best choice in relation to longterm clinical results. Dental lasers have been shown to be effective in treating mild-tomoderate peri-implant lesions in studies with a follow-up mean of 6 months [12]; severe peri-implantitis treatment and longer follow-ups have shown unstable results $[13,14]$. According to some authors, the only non-surgical treatment does not guarantee predictable results in the treatment of mild/moderate peri-implantitis and must be associated with surgical therapy to treat the peri-implant pockets and the bone defects. Non-surgical treatment of mucositis and initial peri-implantitis generally provides clinical improvements in reducing the bleeding on probing and, in some cases, the probing depth.

Early diagnosis and consequent timely intervention remain fundamental elements for the management and success peri-implantitis treatment [33].

Caution is required before interpreting the relevant findings of this study and some of limitations should be borne in mind. First of all, our review comprised studies conducted by several authors with different surgical skills and equipment (instrumentations and biomaterial) and who have chosen different approaches to treat mucositis and peri-implantitis. Furthermore, the study design, the methodology applied in data collecting, the medical and demographic characteristics of the patients treated, the severity of the periodontal disease, the implant surfaces and the duration of the post-operative follow-up periods varied between the analyzed studies.

Consequently, only on the basis of the data reported in the literature, no statistically significant differences were found between the adjunctive laser irradiation and mechanical therapy alone in terms of PPD reduction and CAL gain in peri-implantitis treatment. However, the use of a dental laser to obtain a complete implant surfaces decontamination is convenient to conduce faster intervention and more accepted therapy by the patients. The results collected in our study were strongly influenced by the heterogeneity of the included studies, which present a combination of different therapeutic approach.

For this reason, it is not methodologically possible to establish a single therapeutic strategy to obtain a better implant decontamination in the treatment of peri-implantitis; future research must be encouraged to establish an effective clinical protocol against periimplant lesions.

\section{Conclusions}

Following the results of our meta-analysis, no statistically significant differences was found between laser therapy vs. conventional mechanical therapy in terms of postoperative PPD reduction and CAL gain. However, these results do not invalidate the efficacy of laser irradiation to obtain a valid decontamination of the infected implant parts and of the surrounding hard and soft tissue. Comparative in vivo studies on the efficiency of laser and photodynamic therapy, in addition to conventional therapy, indicate that this technique can induce a significantly higher decontamination of anaerobic bacterial species compared to that obtained with conventional therapy alone. Moreover, the biostimulation inducted by laser irradiation can stimulate a faster peri-implant wound healing.

Diode, Er:YAG and $\mathrm{CO}_{2}$ lasers showed better results, on average, after 6 months of follow-up in the treatment of mucositis and initial peri-implantitis. The longer follow-up 
periods revealed that initial results were unstable, and cases of reinfection were described. However, the relapses that occur in the following months could be more attributable to poor hygienic maintenance by the patient than to an ineffectiveness of the therapy performed.

Author Contributions: Conceptualization, M.C. and M.P.; methodology, M.C. and S.M.; validation, M.P., A.A. and P.S.; investigation, M.P. and M.C.; data curation, A.I.; writing-original draft preparation, P.S., A.A. and S.M.; writing-review and editing, M.C. and M.P.; visualization, A.I.; supervision, M.C.; All authors have read and agreed to the published version of the manuscript.

Funding: This research received no external funding.

Institutional Review Board Statement: Not applicable.

Informed Consent Statement: Not applicable.

Data Availability Statement: Data is contained within the article.

Conflicts of Interest: The authors declare no conflict of interest.

\section{References}

1. Sánchez-Gárces, M.A.; Gay-Escoda, C. Periimplantitis. Med. Oral Patol. Oral Cir. Bucal. 2004, 9, 69-74; 63-69.

2. López-Cerero, L. [Dental implant-related infections]. Enferm. Infecc. Microbiol. Clin. 2008, 26, 589-592. [CrossRef] [PubMed]

3. Algraffee, H.; Borumandi, F.; Cascarini, L. Peri-Implantitis. Br. J. Oral Maxillofac. Surg. 2012, 50, 689-694. [CrossRef]

4. Salmeron, S.; Rezende, M.L.R.; Consolaro, A.; Sant'ana, A.C.P.; Damante, C.A.; Greghi, S.L.A.; Passanezi, E. Laser Therapy as an Effective Method for Implant Surface Decontamination: A Histomorphometric Study in Rats. J. Periodontol. 2013, 84, 641-649. [CrossRef] [PubMed]

5. Lindhe, J.; Meyle, J. Group D of European Workshop on Periodontology Peri-Implant Diseases: Consensus Report of the Sixth European Workshop on Periodontology. J. Clin. Periodontol. 2008, 35, 282-285. [CrossRef]

6. Renvert, S.; Hirooka, H.; Polyzois, I.; Kelekis-Cholakis, A.; Wang, H.-L. Working Group 3 Diagnosis and Non-Surgical Treatment of Peri-Implant Diseases and Maintenance Care of Patients with Dental Implants-Consensus Report of Working Group 3. Int. Dent. J. 2019, 69 (Suppl. 2), 12-17. [CrossRef] [PubMed]

7. Frisch, E.; Ziebolz, D.; Ratka-Krüger, P.; Rinke, S. Double Crown-Retained Maxillary Overdentures: 5-Year Follow-Up. Clin. Implant. Dent. Relat. Res. 2015, 17, 22-31. [CrossRef] [PubMed]

8. Mombelli, A.; Müller, N.; Cionca, N. The Epidemiology of Peri-Implantitis. Clin. Oral Implant. Res. 2012, 23 (Suppl. 6), 67-76. [CrossRef]

9. Heitz-Mayfield, L.J.A.; Mombelli, A. The Therapy of Peri-Implantitis: A Systematic Review. Int. J. Oral Maxillofac. Implant. 2014, 29, 325-345. [CrossRef] [PubMed]

10. Ashnagar, S.; Nowzari, H.; Nokhbatolfoghahaei, H.; Yaghoub Zadeh, B.; Chiniforush, N.; Choukhachi Zadeh, N. Laser Treatment of Peri-Implantitis: A Literature Review. J. Lasers Med. Sci. 2014, 5, 153-162. [PubMed]

11. Tosun, E.; Tasar, F.; Strauss, R.; Kivanc, D.G.; Ungor, C. Comparative Evaluation of Antimicrobial Effects of Er:YAG, Diode, and $\mathrm{CO}_{2}$ Lasers on Titanium Discs: An Experimental Study. J. Oral Maxillofac. Surg. 2012, 70, 1064-1069. [CrossRef] [PubMed]

12. Schwarz, F.; Sculean, A.; Rothamel, D.; Schwenzer, K.; Georg, T.; Becker, J. Clinical Evaluation of an Er:YAG Laser for Nonsurgical Treatment of Peri-Implantitis: A Pilot Study. Clin. Ora.l Implant. Res. 2005, 16, 44-52. [CrossRef] [PubMed]

13. Renvert, S.; Lindahl, C.; Roos Jansåker, A.-M.; Persson, G.R. Treatment of Peri-Implantitis Using an Er:YAG Laser or an Air-Abrasive Device: A Randomized Clinical Trial. J. Clin. Periodontol. 2011, 38, 65-73. [CrossRef]

14. Schwarz, F.; Hegewald, A.; John, G.; Sahm, N.; Becker, J. Four-Year Follow-up of Combined Surgical Therapy of Advanced Peri-Implantitis Evaluating Two Methods of Surface Decontamination. J. Clin. Periodontol. 2013, 40, 962-967. [CrossRef] [PubMed]

15. Pommer, B.; Haas, R.; Mailath-Pokorny, G.; Fürhauser, R.; Watzek, G.; Busenlechner, D.; Müller-Kern, M.; Kloodt, C. Periimplantitis Treatment: Long-Term Comparison of Laser Decontamination and Implantoplasty Surgery. Implant. Dent. 2016, 25, 646-649. [CrossRef] [PubMed]

16. Wang, C.-W.; Ashnagar, S.; Gianflippo, R.D.; Arnett, M.; Kinney, J.; Wang, H.-L. Laser-Assisted Regenerative Surgical Therapy for Peri-Implantitis: A Randomized Controlled Clinical Trial. J. Periodontol. 2020. [CrossRef]

17. Romanos, G.E.; Nentwig, G.H. Regenerative Therapy of Deep Peri-Implant Infrabony Defects after $\mathrm{CO}_{2}$ Laser Implant Surface Decontamination. Int J. Periodontics Restor. Dent. 2008, 28, 245-255.

18. Deppe, H.; Horch, H.-H.; Neff, A. Conventional versus $\mathrm{CO}_{2}$ Laser-Assisted Treatment of Peri-Implant Defects with the Concomitant Use of Pure-Phase Beta-Tricalcium Phosphate: A 5-Year Clinical Report. Int. J. Oral Maxillofac. Implant. 2007, 22, 79-86.

19. Schär, D.; Ramseier, C.A.; Eick, S.; Arweiler, N.B.; Sculean, A.; Salvi, G.E. Anti-Infective Therapy of Peri-Implantitis with Adjunctive Local Drug Delivery or Photodynamic Therapy: Six-Month Outcomes of a Prospective Randomized Clinical Trial. Clin. Oral Implant. Res. 2013, 24, 104-110. [CrossRef] 
20. Bassetti, M.; Schär, D.; Wicki, B.; Eick, S.; Ramseier, C.A.; Arweiler, N.B.; Sculean, A.; Salvi, G.E. Anti-Infective Therapy of PeriImplantitis with Adjunctive Local Drug Delivery or Photodynamic Therapy: 12-Month Outcomes of a Randomized Controlled Clinical Trial. Clin. Oral Implant. Res. 2014, 25, 279-287. [CrossRef]

21. Deppe, H.; Mücke, T.; Wagenpfeil, S.; Kesting, M.; Sculean, A. Nonsurgical Antimicrobial Photodynamic Therapy in Moderate vs Severe Peri-Implant Defects: A Clinical Pilot Study. Quintessence Int. 2013, 44, 609-618. [CrossRef]

22. Bombeccari, G.P.; Guzzi, G.; Gualini, F.; Gualini, S.; Santoro, F.; Spadari, F. Photodynamic Therapy to Treat Periimplantitis. Implant. Dent. 2013, 22, 631-638. [CrossRef] [PubMed]

23. Papadopoulos, C.A.; Vouros, I.; Menexes, G.; Konstantinidis, A. The Utilization of a Diode Laser in the Surgical Treatment of Peri-Implantitis. A Randomized Clinical Trial. Clin. Oral Investig. 2015, 19, 1851-1860. [CrossRef] [PubMed]

24. Tenore, G.; Montori, A.; Mohsen, A.; Mattarelli, G.; Palaia, G.; Romeo, U. Evaluation of Adjunctive Efficacy of Diode Laser in the Treatment of Peri-Implant Mucositis: A Randomized Clinical Trial. Lasers Med. Sci. 2020, 35, 1411-1417. [CrossRef]

25. Mettraux, G.R.; Sculean, A.; Bürgin, W.B.; Salvi, G.E. Two-Year Clinical Outcomes Following Non-Surgical Mechanical Therapy of Peri-Implantitis with Adjunctive Diode Laser Application. Clin. Oral Implant. Res. 2016, 27, 845-849. [CrossRef]

26. Kotsovilis, S.; Karoussis, I.K.; Trianti, M.; Fourmousis, I. Therapy of Peri-Implantitis: A Systematic Review. J. Clin. Periodontol. 2008, 35, 621-629. [CrossRef] [PubMed]

27. Giannelli, M.; Lasagni, M.; Bani, D. Thermal Effects of $\lambda=808 \mathrm{Nm}$ GaAlAs Diode Laser Irradiation on Different Titanium Surfaces. Lasers Med. Sci. 2015, 30, 2341-2352. [CrossRef]

28. Kreisler, M.; Götz, H.; Duschner, H. Effect of Nd:YAG, Ho:YAG, Er:YAG, CO 2, and GaAIAs Laser Irradiation on Surface Properties of Endosseous Dental Implants. Int. J. Oral Maxillofac. Implant. 2002, 17, $202-211$.

29. Ganz, C.H. Evaluation of the Safety of the Carbon Dioxide Laser Used in Conjunction with Root Form Implants: A Pilot Study. J. Prosthet. Dent. 1994, 71, 27-30. [CrossRef]

30. Matsuyama, T.; Aoki, A.; Oda, S.; Yoneyama, T.; Ishikawa, I. Effects of the Er:YAG Laser Irradiation on Titanium Implant Materials and Contaminated Implant Abutment Surfaces. J. Clin. Laser Med. Surg. 2003, 21, 7-17. [CrossRef] [PubMed]

31. Taniguchi, Y.; Aoki, A.; Mizutani, K.; Takeuchi, Y.; Ichinose, S.; Takasaki, A.A.; Schwarz, F.; Izumi, Y. Optimal Er:YAG Laser Irradiation Parameters for Debridement of Microstructured Fixture Surfaces of Titanium Dental Implants. Lasers Med. Sci. 2013, 28, 1057-1068. [CrossRef] [PubMed]

32. Aoki, A.; Mizutani, K.; Schwarz, F.; Sculean, A.; Yukna, R.A.; Takasaki, A.A.; Romanos, G.E.; Taniguchi, Y.; Sasaki, K.M.; Zeredo, J.L.; et al. Periodontal and Peri-Implant Wound Healing Following Laser Therapy. Periodontology 2000 2015, 68, $217-269$. [CrossRef] [PubMed]

33. Wang, C.-W.; Renvert, S.; Wang, H.-L. Nonsurgical Treatment of Periimplantitis. Implant. Dent. 2019, 28, 155-160. [CrossRef] 